\title{
Analysis of correlation between HP infection and activation of PI3K/Akt pathway in mucosal tissues of gastric cancer and precancerous lesions
}

\author{
YI XIE ${ }^{1}$ and $\mathrm{LI} \mathrm{LIU}^{2}$ \\ Departments of ${ }^{1}$ Gastroenterology and ${ }^{2}$ Oncology, The Fifth People's Hospital of Chongqing, Chongqing 400062, P.R. China
}

Received December 1, 2017; Accepted May 2, 2018

DOI: $10.3892 / \mathrm{ol} .2018 .9329$

\begin{abstract}
The aim of the study was to investigate the correlation between Helicobacter pylori (HP) infection and activation of the phosphatidylinositol-3-kinase/Akt (PI3K/ $\mathrm{Akt}$ ) pathway in mucosal tissues of gastric cancer and precancerous lesions. Patients with chronic atrophic gastritis $(n=52)$ and gastric cancer $(n=98)$ were treated in the Department of Gastroenterology at The Fifth People's Hospital of Chongqing from August 2011 to August 2016 were selected, and the biopsy tissue and serum specimens were collected. The HP infection was detected via enzyme-linked immunosorbent assay (ELISA), and the expression level of phosphorylatedAkt (p-Akt) was detected via immunohistochemistry (IHC). Moreover, in vivo experiments were performed to simulate HP infection in gastric cancer cells (MGC-803 and AGS), and the p-Akt protein level, PI3K activity and cell proliferative activity were detected. Finally, the changes in Akt protein level were detected by co-culture of gastric cancer cells via LY294002, a PI3K inhibitor, and HP. The positive rate of HP infection in patients with chronic atrophic gastritis was $84.6 \%$ (44/52), which was significantly higher than that in patients with gastric cancer [73.5\% (72/98)] $(\mathrm{p}<0.05)$. The positive rate of HP infection in patients with early gastric cancer $(86.4 \%)$ was significantly higher than that in patients with moderate-advanced gastric cancer $(69.7 \%) \quad(\mathrm{p}<0.05)$. Results of IHC and western blot analysis revealed that the p-Akt expression level in HP-positive tissues was obviously higher than that in HP-negative tissues $(\mathrm{p}<0.05)$. In vitro cell experiments revealed that the PI3K activity was enhanced and the PI3K/Akt pathway was significantly activated after HP infection in tumor cells, thus promoting the proliferation of tumor cells $(\mathrm{p}<0.05)$ in a time-dependent manner. After LY294002 inhibited PI3K activity, Akt was not significantly activated by HP infection. Thus, HP activates the PI3K/Akt
\end{abstract}

Correspondence to: Dr Li Liu, Department of Oncology, The Fifth People's Hospital of Chongqing, 24 Renji Road, Nan'an, Chongqing 400062, P.R. China

E-mail: youbang1011@163.com

Key words: gastric cancer, Helicobacter pylori, PI3K/Akt, p-Akt pathway in gastric cancer cells, thereby promoting tumor cell proliferation.

\section{Introduction}

Gastric cancer is the most common malignant tumor in the digestive tract. In China, over 160,000 individuals succumb to gastric cancer annually, accounting for approximately one fifth of all tumor deaths, posing a serious threat to human health $(1,2)$. It has been shown that both bacterial and host genetic factors affect the progression of gastric diseases with individual differences. The Helicobacter pylori (HP) strain is a main pathogenic factor, whose toxic areas are fatal pathogenicity island (PAI) and vacuolatingcytotoxin (VacA) $(3,4)$. In $10-20 \%$ infected patients, HP induces chronic gastritis to develop into gastroduodenal ulcer, gastric cancer or gastric mucosa-associated lymphoid tissue lymphoma $(5,6)$. Gastric precancerous lesions refer to histopathological changes in gastric mucosa, namely the gastric mucosa dysplasia and intestinal metaplasia, which are prone to cancerization (7).

Phosphatidylinositol-3-kinase (PI3K) signals are involved in cell proliferation, differentiation, apoptosis, and glucose transport (8). After PI3K activation, a second messenger, phosphatidylinositol triphosphate (PIP3), is generated on the cytoplasmic membrane, which binds to the signal proteins, Akt and phosphoinositide-dependent kinase 1 (PDK1), containing the $\mathrm{PH}$ structural domain in cells, leading to Akt activation (9). Akt, also known as protein kinase B (PKB), is the main downstream effector of PI3K. The phosphorylation of Ser473 and Thr308 sites is a necessary condition for Akt activation, and the activated Akt further phosphorylates or inhibits its downstream target proteins, such as glycogen synthase kinase-3 (GSK-3), glucose transporter (GLUT), mammalian target of rapamycin (mTOR), caspase-9 and nuclear factor $-\kappa \mathrm{B}(\mathrm{NF}-\kappa \mathrm{B})$, thus regulating the cell proliferation, differentiation, apoptosis and migration $(10,11)$. However, there are few reports on the correlation between HP infection and PI3K/Akt pathway activation in mucosal tissues of gastric cancer and precancerous lesions.

In this study, the correlation between HP infection and PI3K/Akt pathway activation in mucosal tissues of gastric cancer and precancerous lesions was analyzed, providing strong evidence for the clinical treatment of chronic gastritis and application of anti-inflammatory drugs in gastric cancer. 


\section{Materials and methods}

General data. Patients with chronic atrophic gastritis $(\mathrm{n}=52)$ and gastric cancer $(n=98)$ treated at the Department of Gastroenterology at at The Fifth People's Hospital of Chongqing (Chongqing, China) from August 2010 to August 2016 were selected. Patients were diagnosed via gastroscopy and pathological examination. The biopsy tissue and serum specimens were collected, and tissues were fixed via $4 \%$ neutral formalin, embedded in paraffin, and serially sectioned $(4 \mu \mathrm{m})$ for immunohistochemistry (IHC). None of patients received radio- and/or chemotherapy. There was no history of taking anti-HP drugs or non-steroidal anti-inflammatory drugs within 2 weeks before gastroscopy, and patients with other systemic malignancies were excluded. Early gastric cancer was defined as cancer tissue infiltration in mucosal and submucosal layers. Moderate-advanced or progressive gastric cancer was defined as invasion of cancer tissues into the gastric muscular wall and serosal layer. The study was approved by the Ethics Committee of The Fifth People's Hospital of Chongqing and written informed consents were signed by the patients and/or guardians.

Enzyme-linked immunosorbent assay (ELISA). Whole blood was collected and centrifuged at $600 \mathrm{x} \mathrm{g}$ for $10 \mathrm{~min}$ to separate the serum. The PI3K activity assay kit (Art. No. K-1000S, Echelon, New York, NY, USA) was used. According to the instructions provided, the serum was added, and $50 \mu \mathrm{l}$ enzyme conjugate was also added into each well, except the control well. The mixture was mixed evenly and incubated at $37^{\circ} \mathrm{C}$ for $30 \mathrm{~min}$, and the supernatant was discarded. The wells were washed with washing liquid 5 times, and $50 \mu \mathrm{l}$ color developing agents A and B (1:1) were added into each well, mixed evenly and incubated in the dark at $37^{\circ} \mathrm{C}$ for $15 \mathrm{~min}$. Then, $50 \mu \mathrm{l}$ stop buffer was added into each well to terminate the immune reaction, and the results were read at the wavelength of $450 \mathrm{~nm}$ using a microplate reader (Bio-Rad Laboratories, Inc., Hercules, CA, USA). HP immunoglobulin G ( $\operatorname{IgG})$ $>20 \mathrm{U} / \mathrm{ml}$ indicated a positive result.

IHC. Paraffin-embedded tissue sections were taken and used for IHC. After dewaxing via xylene, dehydration via gradient ethanol, and antigen retrieval via sodium citrate buffer solution in a microwave were carried out, $3 \% \mathrm{H}_{2} \mathrm{O}_{2}$ blocker was added to block the peroxidase. Sections were sealed in $10 \%$ donkey serum, added with the primary antibody (total p-Akt, Abcam, Cambridge, MA, USA, diluted at 1:500), and placed in a wet box for incubation at $4^{\circ} \mathrm{C}$ overnight. The following day, the sections were washed with phosphate-buffered saline (PBS) three times, and the ready-to-use universal secondary antibody was incubated, followed by color development via diaminobenzidine $(\mathrm{DAB})$ and capturing of images under a microscope.

Western blotting. Four cases of HP-positive and 4 cases of HP-negative gastric cancer tissues were randomly selected and ground to extract the proteins using IP lysis (with cocktail). The protein concentration was detected via bicinchoninic acid (BCA); the protein was separated via $12 \%$ polyacrylamide gel electrophoresis, transferred onto the polyvinylidene fluoride (PVDF) membrane via semi-dry process, and sealed with 5\% skimmed milk powder at room temperature for $1 \mathrm{~h}$. The bands were incubated with rabbit anti-human primary monoclonal antibodies, p-Akt Ser473 (1:2,000), p-Akt Thr308 (1:1,000), total p-Akt $(1: 2,000)$ and $\beta$-actin $(1: 5,000)$ (cat. nos. 4060, 13038, 4685, 8457, respectively; Cell Signaling Technology, Danvers, MA, USA) at $4^{\circ} \mathrm{C}$ overnight. The following day, the membrane was washed with PBST three times (10 min/time). The secondary goat anti-rabbit polyclonal antibody $(1: 2,000$; cat. no. 7074; Cell Signaling Technology) was added for incubation at room temperature for $2 \mathrm{~h}$, and the membrane was washed again with PBST three times (10 min/time). Then the hypersensitive luminescent solution was added, and images were captured using a gel imaging system.

HP infection. HP strains were purchased from the American Type Culture Collection (Shanghai, China). Gastric cancer cell lines MGC-803 and AGS were cultured with RPMI-1640 and $10 \%$ fetal bovine serum. Bacterial liquid was added into the medium for co-culture, and the proteins were extracted at 0,6 and $12 \mathrm{~h}$. The p-Akt protein level was detected via western blot analysis.

Statistical analysis. MedCalc software (Marie-Kerque, Belgium) was used for data statistics and processing. Measurement data were presented as mean \pm standard deviation. ANOVA was used for comparison between multiple groups and the post hoc test was SNK test. The $\chi^{2}$ test was used for the comparison of enumeration data. $\mathrm{P}<0.05$ indicated the difference was statistically significant.

\section{Results}

Comparison of positive rate of HP infection between patients with precancerous lesions and those with gastric cancer. The serum HP infections in patients with precancerous lesions and gastric cancer were detected via ELISA (Table I). The positive rate of HP infection in patients with precancerous lesions was $84.6 \%$ (44/52), which was significantly higher than that in patients with gastric cancer [73.5\% (72/98)] $(\mathrm{p}<0.05)$. The positive rate of HP infection in patients with early gastric cancer $(86.4 \%)$ was significantly higher than that in patients with moderate-advanced gastric cancer $(69.7 \%)(\mathrm{p}<0.05)$.

Detection of $p$-Akt levels in both groups via IHC. P-Akt was mainly located in the cytoplasm, and the p-Akt expression level in HP-positive tissues was obviously higher than that in HP-negative tissues $(\mathrm{p}<0.05)$ (Fig. 1).

Detection of $p$-Akt protein level via western blot analysis. Four cases of HP-positive and 4 cases of HP-negative gastric cancer tissues were randomly selected to detect the Akt phosphorylation level via western blotting. The p-Akt expression level was high in HP-positive tissues, but low in HP-negative tissues (Fig. 2).

Analysis of correlation between HP infection and PI3K activity. Total proteins were extracted from cells at 0,6 and $12 \mathrm{~h}$ after HP infection, and the PI3K activity was detected using the PI3K activity assay kit. PI3K activity was significantly enhanced after HP infection (p<0.05) (Fig. 3). 
Table I. Comparison of positive rate of HP infection between patients with precancerous lesions and those with gastric cancer.

\begin{tabular}{lccccc}
\hline & & \multicolumn{3}{c}{ HP infection } \\
\cline { 3 - 5 } Variables & No. & Negative & Positive & Positive rate (\%) & $\chi^{2}$ \\
\hline Chronic atrophic gastritis & 52 & 8 & 44 & 84.6 & 5.782 \\
Gastric cancer & 98 & 26 & 72 & 73.5 & $0.012^{\mathrm{a}}$ \\
Early & 22 & 3 & 19 & 86.4 & $0.026^{\mathrm{b}}$ \\
Advanced & 76 & 23 & 53 & 69.7 & 4.265 \\
\hline
\end{tabular}

Gastric cancer vs. precancerous lesions, ${ }^{\mathrm{a}} \mathrm{p}<0.05$; early gastric cancer vs. advanced gastric cancer, ${ }^{\mathrm{b}} \mathrm{p}<0.05$.

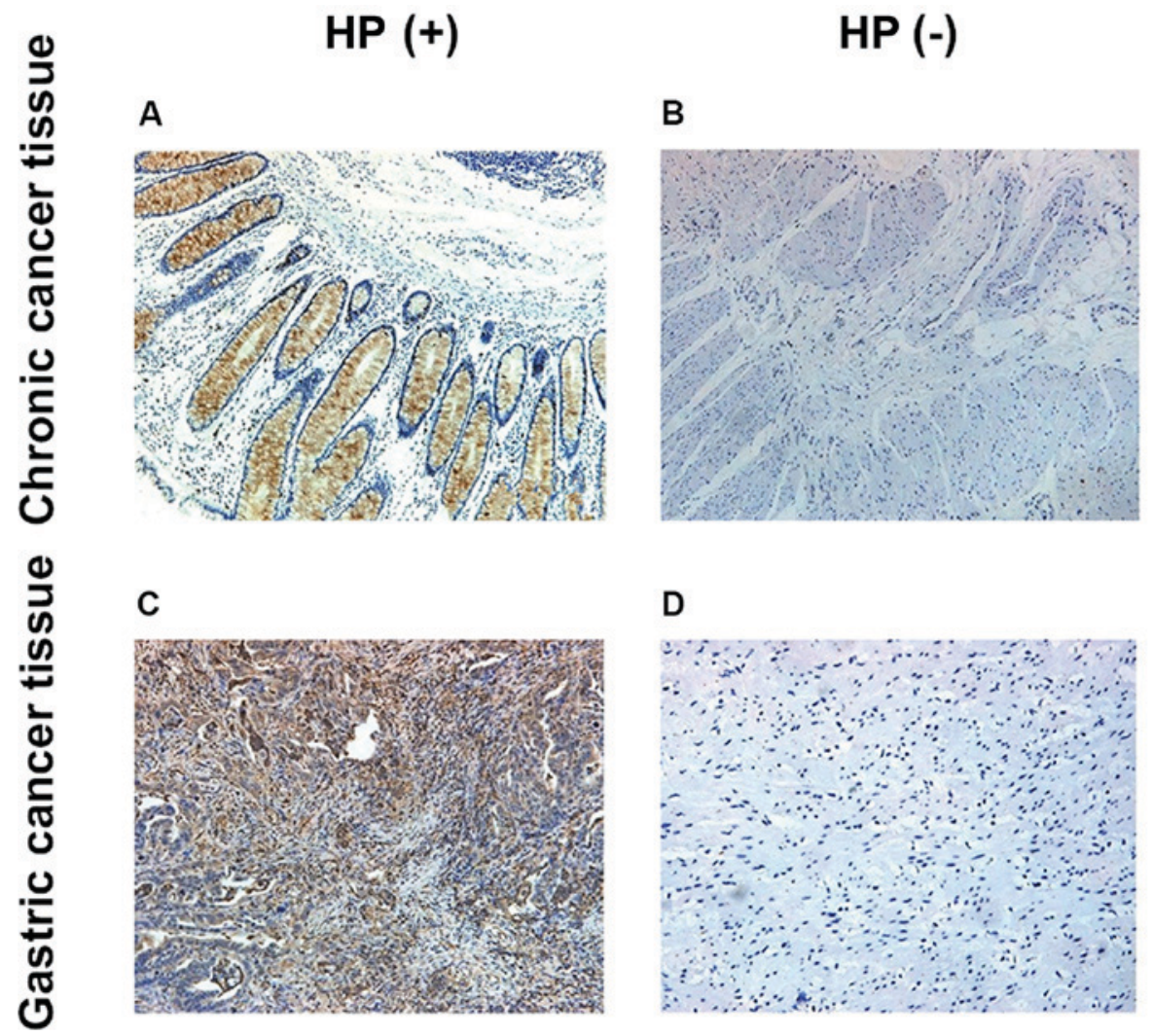

Figure 1. Detection of Akt phosphorylation levels in chronic gastritis and gastric cancer tissues via IHC. (A) Positive expression of Akt protein in HP positive gastric mucosa. (B) Negative expression of Akt protein in HP negative gastric mucosa. (C) Positive expression of Akt protein in HP positive gastric cancer tissue. (D) Negative expression of Akt protein in HP negative gastric cancer tissue. HP, Helicobacter pylori.

A

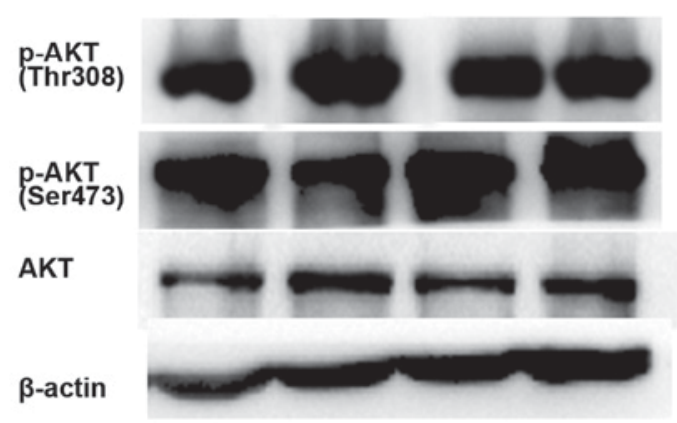

B

HP (-)

Figure 2. Detection of p-Akt protein expression level in clinical tissues via western blotting. Expression of total Akt protein and phosphorylation of Thr308 and ser473 sites in (A) HP positive and (B) HP negative gastric cancer tissues by Western blot analysis. HP, Helicobacter pylori. 
A

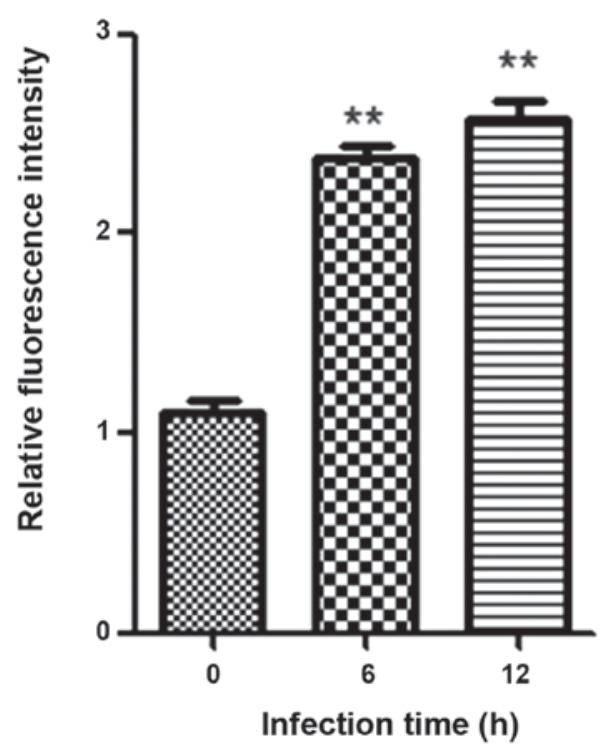

B

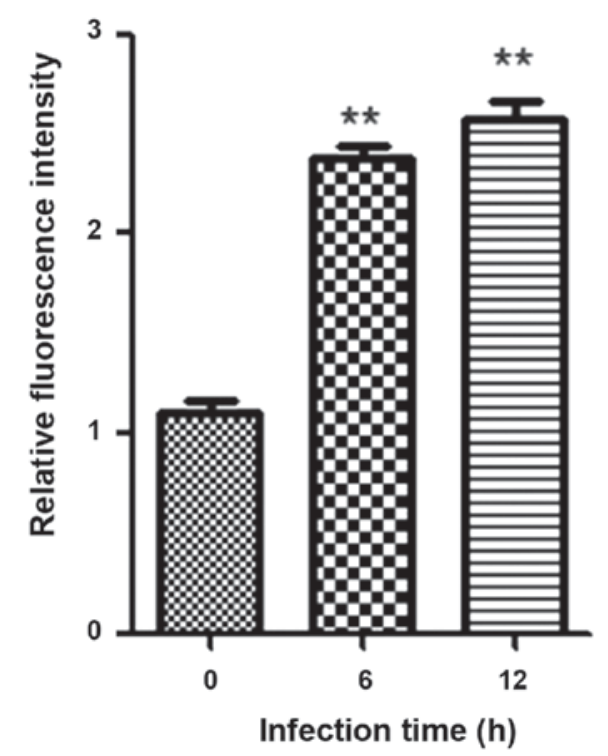

Figure 3. Changes in PI3K activity at 0,6 and $12 \mathrm{~h}$ after HP infection of gastric cancer cells. Compared with that at $0 \mathrm{~h},{ }^{* * *} \mathrm{p}<0.01$. The activity of PI3K enzyme increased with time after HP infection of gastric carcinoma (A) MGC-803 cells and (B) AGS cells. HP, Helicobacter pylori.

A

MGC-803
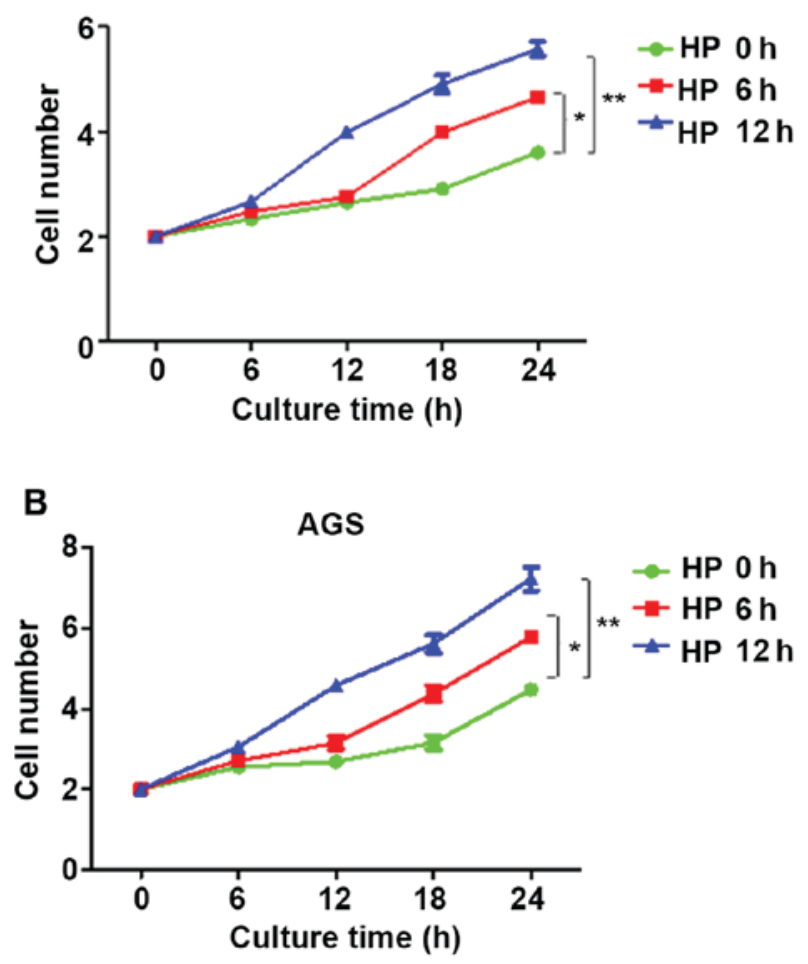

Figure 4. Correlation between HP infection and gastric cancer cell proliferation. The cell count method showed that the proliferation rate of (A) MGC-803 and (B) AGS cells after HP infection was significantly increased, and showed a time-dependent trend. ${ }^{*} \mathrm{p}<0.05 ;{ }^{* *} \mathrm{p}<0.01$. HP, Helicobacter pylori.

Analysis of correlation between HP infection and cell proliferation. At 0,6 and $12 \mathrm{~h}$ after HP infection of cells, the infection medium was discarded. The cells were digested and

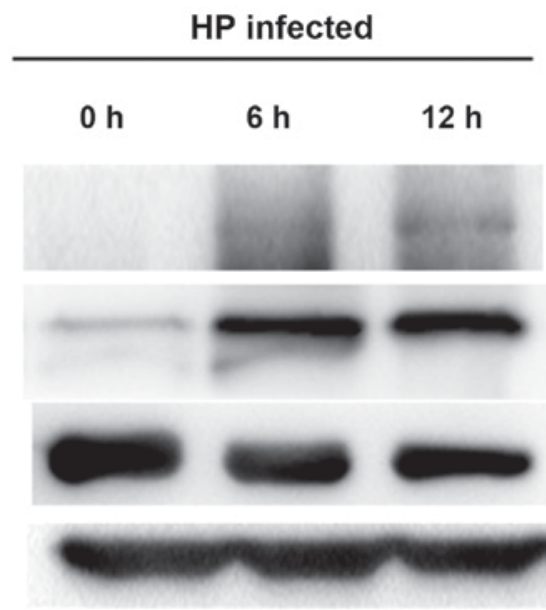

Figure 5. Changes in p-Akt protein levels after HP infection in gastric cancer cells. HP, Helicobacter pylori.

inoculated in a 96-well plate. The number of cells at 4, 8, 12, 16,20 and $24 \mathrm{~h}$ of culture after HP infection was counted using the cell counting method. Compared with that at $0 \mathrm{~h}$, the proliferation rates of cells at $6 \mathrm{~h}$ and $12 \mathrm{~h}$ after HP infection were significantly increased in a time-dependent manner $(\mathrm{p}<0.05)$ (Fig. 4).

Detection of correlation between HP infection and p-Akt via in vitro experiments. The gastric cancer MGC-803 and AGS cells were co-cultured with HP. At 0,6 and $12 \mathrm{~h}$ after HP infection of cells, the protein was extracted from cells to detect the p-Akt expression via western blotting. The results showed that the p-Akt expressions (Ser473 and Thr308) in cells after HP infection were significantly increased $(p<0.05)$; with the passage of time, the Akt phosphorylation level was gradually elevated in a time-dependent manner (Fig. 5). 


\section{HP infected}

$0 \mathrm{~h} \quad 6 \mathrm{~h} \quad 12 \mathrm{~h}$

\section{p-AKT Thr308}

p-AKT Ser473

Total AKT

$\beta$-actin

Figure 6. Detection of correlation between HP infection and p-Akt. HP, Helicobacter pylori.

Detection of correlation between HP infection and p-Akt. After LY294002, a PI3K inhibitor, was added, the PI3K activity was suppressed, thus inhibiting the Akt activation, namely the Akt phosphorylation. Therefore, LY294002 was added into the cell culture solution for co-culture with HP. The protein was extracted to detect the p-Akt expression level. Akt was not significantly activated by HP infection after inhibition of PI3K activity (Fig. 6).

\section{Discussion}

At present, the pathogenesis of gastric cancer is not yet fully clear, and the implementation of etiological primary prevention is more difficult; thus, research on gastric precancerous lesions is crucial in the secondary prevention of gastric cancer. Early identification, prevention and treatment of precancerous diseases and lesions have become effective methods used to reduce the incidence and mortality rates of gastric cancer (12).

In tumor cells, the Akt/PI3K pathway is abnormally activated. Activated Akt phosphorylates a large number of downstream substrates and promotes the upregulation of cell proliferation. Moreover, Akt directly promotes cell survival through phosphorylation and the inactivation of several pro-apoptotic targets (including Bad, Bim, Bax and FoxO1/3a transcription factors) (13). In addition, Akt also plays an important role in metabolism, which regulates glycolysis through phosphorylation of phosphofructokinase (PFK) and hexokinase, and exerts important functions in the anaerobic glycolysis (also known as the Warburg effect) of cancer cells (14). The activation of lipid kinase PI3K can activate Akt, thus generating PIP3 in the cytoplasmic membrane. Akt binds to PIP3 through its substrate protein homology $(\mathrm{PH})$ domain, resulting in the translocation of Akt to the cell membrane. Akt can be activated via dual phosphorylation mechanism. PDK1 is also translocated to the cytoplasmic membrane due to its own PH domain, which can phosphorylate Akt at the Thr308 site in the activation loop $(15,16)$. The secondary phosphorylation of carboxy-terminal Ser473 site of Akt is completed by the mTOR-Rictor complex, mTORC2, which is also necessary for Akt activation (17).
A large number of studies have shown that HP infection is closely related to the occurrence and development of gastric cancer. Currently, HP infection can be clinically diagnosed via endoscopy and breath test (18). Tabassam et al found that HP virulence factors, cag PAI and OipA, regulate the phosphorylation of Akt sites, Thr308 and Ser473, respectively. OipA mutation reduces the phosphorylation level of Akt Ser473, whereas HP infection-induced cag PAI mutation reduces the activation of Akt Thr308. The specific function of cag PAI or OipA in activating the signal transduction in Akt Ser473 or Thr308 may lead to the imbalance of downstream proliferation and apoptosis signaling (11). Importantly, infection with the cag PAI/OipA double mutants completely blocks the activation of two HP-mediated Akt sites, suggesting that both OipA and cag PAI are necessary for the complete activation of Akt (19). Activated Akt signaling pathway is a key regulator in many cellular biological effects, such as cell survival, proliferation and motility (20). The upregulation of Akt activation is also observed in tissues adjacent to gastric tumors, and the activation of Akt affects the chemoresistance of gastric cancer (21). In summary, the phosphorylation of Akt mediated jointly by cag PAI and OipA is thought to be an intracellular signaling regulator of occurrence of gastric cancer, as well as a key regulator of many cellular functions.

In the present study, serum specimens of patients with chronic gastritis and gastric cancer were collected. The serum HP level was detected via ELISA to further confirm whether the phosphorylation level of Akt could be significantly upregulated after HP infection in patients with gastritis or gastric cancer, namely the PI3K/Akt pathway activation, thereby promoting the tumor occurrence and development. At the same time, biopsy tissue specimens of patients were collected. The p-Akt level, namely the PI3K/Akt pathway activation, in tissues was detected via IHC. In in vitro experiments, gastric cancer MGC-803 and AGS cells were co-cultured with HP, and it was found that both PI3K activity and p-Akt protein level were significantly increased after cell infection. Moreover, the cell count experiments showed that HP infection could significantly increase the proliferative activity of gastric cancer cells. In addition, the co-culture of LY294002 and HP revealed that there was no significant change in the p-Akt protein level. In conclusion, it is proved in this study through clinical cases combined with in vitro experiments that the PI3K/Akt pathway can be activated after HP infection, thereby promoting the occurrence and development of tumors.

\section{Acknowledgements}

Not applicable.

\section{Funding}

No funding was received.

\section{Availability of data and materials}

The datasets used and/or analyzed during the current study are available from the corresponding author on reasonable request. 


\section{Authors' contributions}

YX collected the general data of patients. YX and LL were responsible for IHC and western blot analysis. Both authors read and approved the final manuscript.

\section{Ethics approval and consent to participate}

The study was approved by the Ethics Committee of The Fifth People's Hospital of Chongqing (Chongqing, China) and written informed consents were signed by the patients and/or guardians.

\section{Patient consent for publication}

Not applicable.

\section{Competing interests}

The authors declare that they have no competing interests.

\section{References}

1. Smith MG, Hold GL, Tahara E and El-Omar EM: Cellular and molecular aspects of gastric cancer. World J Gastroenterol 12: 2979-2990, 2006.

2. Gotoda T, Yanagisawa A, Sasako M, Ono H, Nakanishi Y, Shimoda $\mathrm{T}$ and Kato Y: Incidence of lymph node metastasis from early gastric cancer: Estimation with a large number of cases at two large centers. Gastric Cancer 3: 219-225, 2000.

3. Kim SS, Ruiz VE, Carroll JD and Moss SF: Helicobacter pylori in the pathogenesis of gastric cancer and gastric lymphoma. Cancer Lett 305: 228-238, 2011.

4. Moss SF and Sood S: Helicobacter pylori. Curr Opin Infect Dis 16: 445-451, 2003.

5. Tammer I, Brandt S, Hartig R, König W and Backert S: Activation of Abl by Helicobacter pylori: A novel kinase for CagA and crucial mediator of host cell scattering. Gastroenterology 132: $1309-1319,2007$.

6. Murata-Kamiya N, Kurashima Y, Teishikata Y, Yamahashi Y, Saito Y, Higashi H, Aburatani H, Akiyama T, Peek RM Jr, Azuma $\mathrm{T}$, et al: Helicobacter pylori CagA interacts with E-cadherin and deregulates the $\beta$-catenin signal that promotes intestinal transdifferentiation in gastric epithelial cells. Oncogene 26: 4617-4626, 2007.

7. de Vries AC, van Grieken NCT, Looman CWN, Casparie MK, de Vries E, Meijer GA and Kuipers EJ: Gastric cancer risk in patients with premalignant gastric lesions: A nationwide cohort study in the Netherlands. Gastroenterology 134: 945-952, 2008.
8. Thorpe LM, Yuzugullu $\mathrm{H}$ and Zhao JJ: PI3K in cancer: Divergent roles of isoforms, modes of activation and therapeutic targeting. Nat Rev Cancer 15: 7-24, 2015.

9. Liang J and Slingerland JM: Multiple roles of the PI3K/PKB (Akt) pathway in cell cycle progression. Cell Cycle 2: 339-345, 2003.

10. Hers I, Vincent EE and Tavaré JM: Akt signalling in health and disease. Cell Signal 23: 1515-1527, 2011

11. Tabassam FH, Graham DY and Yamaoka Y: Helicobacter pylori activate epidermal growth factor receptor- and phosphatidylinositol 3-OH kinase-dependent Akt and glycogen synthase kinase $3 \beta$ phosphorylation. Cell Microbiol 11: 70-82, 2009.

12. Oda I, Saito D, Tada M, Iishi H, Tanabe S, Oyama T, Doi T, Otani Y, Fujisaki J, Ajioka Y, et al: A multicenter retrospective study of endoscopic resection for early gastric cancer. Gastric Cancer 9: 262-270, 2006.

13. Li W, Wang H, Kuang CY, Zhu JK, Yu Y, Qin ZX, Liu J and Huang L: An essential role for the Id1/PI3K/Akt/NFkB/survivin signalling pathway in promoting the proliferation of endothelial progenitor cells in vitro. Mol Cell Biochem 363: 135-145, 2012.

14. Kurup PA: Endosymbioticactinidicarchaeal mediated warburg phenotype mediates human disease state. Adv Nat Sci 5: 81-84, 2012.

15. Chappell WH, Steelman LS, Long JM, Kempf RC, Abrams SL, Franklin RA, Bäsecke J, Stivala F, Donia M, Fagone P, et al: Ras/Raf/MEK/ERK and PI3K/PTEN/Akt/mTOR inhibitors: Rationale and importance to inhibiting these pathways in human health. Oncotarget 2: 135-164, 2011.

16. Bitting RL and Armstrong AJ: Targeting the PI3K/Akt/mTOR pathway in castration-resistant prostate cancer. Endocr Relat Cancer 20: R83-R99, 2013.

17. Bayascas JR and Alessi DR: Regulation of Akt/PKB Ser473 phosphorylation. Mol Cell 18: 143-145, 2005.

18. Wong BCY, Lam SK, Wong WM, Chen JS, Zheng TT, Feng RE, Lai KC, Hu WH, Yuen ST, Leung SY, et al; China Gastric Cancer Study Group: Helicobacter pylori eradication to prevent gastric cancer in a high-risk region of China: A randomized controlled trial. JAMA 291: 187-194, 2004.

19. Wei J, Nagy T A, Vilgelm A, Zaika E, Ogden SR, Romero-Gallo J, Piazuelo MB, Correa P, Washington MK, El-Rifai W, et al: Regulation of p53 tumor suppressor by Helicobacter pylori in gastric epithelial cells. Gastroenterology 139: 1333-1343, 2010.

20. Hu Y, Qiao L, Wang S, Rong SB, Meuillet EJ, Berggren M, Gallegos A, Powis G and Kozikowski AP: 3-(Hydroxymethyl)bearing phosphatidylinositol ether lipid analogues and carbonate surrogates block PI3-K, Akt, and cancer cell growth. J Med Chem 43: 3045-3051, 2000.

21. Page C, Lin HJ, Jin Y, Castle VP, Nunez G, Huang M and Lin J: Overexpression of Akt/AKT can modulate chemotherapyinduced apoptosis. Anticancer Res 20A: 407-416, 2000.

c) (i) $\ominus$ This work is licensed under a Creative Commons

CY NC ND Attribution-NonCommercial-NoDerivatives 4.0 International (CC BY-NC-ND 4.0) License. 